\title{
Size effects in metal foam cores for sandwich structures
}

\author{
Joseph F. Rakow and Anthony M. Waas * \\ Composite Structures Laboratory, \\ Department of Aerospace Engineering, \\ University of Michigan, Ann Arbor, MI 48109-2140
}

February 5, 2003

\begin{abstract}
The shear response of aluminum foam, including size effects, is measured and quantified for a closed cell aluminum foam. The shear stiffness is shown to depend linearly on density, while the strength exhibits a power law dependence. The linear response is shown to be independent of strain rate for low rate dynamic loading, while the strength and energy absorption increase with increasing strain rate. The density dependence of the stiffness is reproduced analytically based on the composite cylinders model. Optical techniques are used to measure the strain field of the experimental specimens throughout the loading program. By evaluating concentric sub-regions of the sample, a sample size of 18 mean cell diameters is determined to be the dimension below which the uncertainty in the predicted shear modulus of an aluminum foam sample increases significantly. This length scale threshold is replicated in a periodic finite element structure with randomly distributed imperfections.
\end{abstract}

\section{Introduction}

Metal foams represent an attractive alternative for sandwich structure cores for multiple reasons. With metal foam cores, the adhesive substrate of a sandwich structure may be eliminated with in-production integral bonding to metallic face sheets, stiffening the sandwich and broadening its range of operating environments [1]. Metal foams exhibit a compressive stress-strain response that is ideal for energy absorption and impact alleviation with a long, constant stress, plastic strain plateau

\footnotetext{
* Graduate Research Assistant and Professor of Aerospace Engineering, respectively, University of Michigan. Assoc. Fellow AIAA, Copyright (C) 2003 by Anthony M. Waas. Published by the AIAA, with permission.
}

[2]. An open cell metal foam offers an opportunity to eliminate the catastrophic nature of water or cryogenic gas permeation that has crippled the long-term use of sandwich constructions with honeycomb cores [3]. An open cell construction also allows for active cooling of the sandwich structure, elevating its range of acceptable operating temperatures.

For integration into sandwich structures, the shear behavior of metal foam must be understood. Some disparate results regarding shear behavior currently exist in the literature. One study found a linear relationship between shear strength and density [4], while a cubic lattice model subjected to shear loading predicted a non-linear power law dependence [5]. Another investigation offers only a few data points for shear stiffness and strength of melt-foamed aluminum [6]. Furthermore, these experiments involved thin specimens, with no account for size effects.

The present paper offers the full shear response curves for a broad range of density. The density dependence of stiffness and strength are found experimentally with the former being reproduced analytically. The strain rate dependence of the shear response is also considered for low-rate dynamic loading. The effect of specimen size, relative to the mean cell size, is analyzed experimentally with a unique approach involving digital image correlation. The observed behavior is reproduced with a finite element model. These analyses identify a threshold in the ratio of specimen size to cell size below which the shear response of a given sample is associated with a significant amount of uncertainty. 


\section{Shear Response - Experi- ments}

The details of the experimental procedure and an extended analysis of the results are presented in [7]; a summary is presented here. Square samples of $\mathrm{SiC}$ stabilized aluminum foam, produced by Cymat (Ontario, Canada), were subjected to shear loading in a window frame device. The frame, shown in Figure 1, is similar to that suggested by ASTM C273, but modified to avoid through the thickness size effects, such that bulk properties may be measured. Samples ranging in density over $4-15 \%$ were tested to obtain the density-dependent relationships for the mechanical properties. Strain rate effects were studied through the low-rate dynamic regime covering the range of $3.65 E-5 / s$ to $0.17 / s$.

A representative response curve, up to and beyond the maximum load, is shown in Figure 2. The material exhibits a linear region leading to a peak load and a subsequent dramatic loss of load carrying capability, as large visible fractures form at the peak load. The post-linear response of aluminum foam in shear is markedly different from its uniaxial response, which exhibits a long constant stress plateau after peak load, leading to densification and a further increase in load carrying capability [2]. The early onset of plastic deformation, illustrated by the unloading response of Figure 2 is typical of all metal foam deformation [5].

The strain rate dependence of the shear response for static and low-rate dynamic loading is shown in Figure 3 for two samples of like density. The linear response is clearly independent of strain rate, while the strength and energy absorption show a slight increase. On average, the samples subjected to dynamic loading had a specific strength $24 \%$ greater and specific energy absorption $36 \%$ greater than equivalent samples under static loading.

Figure 4 and Figure 5 show the density dependence and strain rate dependence of the modulus and strength, respectively, of aluminum foam in shear. The modulus follows a linear dependence on density and is sufficiently lower than the lower bound of a model presented in [5]. The static strength follows a power law dependence as predicted in [5]. For a complete understanding of the shear strain rate dependence of aluminum foam, the methods employed in the present work must be extended to higher strain rates via, for example, drop tower and split Hopkinson bar experiments.

The sensitivity of aluminum foam to compressive and tensile strain rates have been studied in [8]-
[12]. Static experiments on the uniaxial behavior of aluminum foams were conducted in [4],[13]-[16]. [4] and [6] measured the static shear properties of some aluminum foam samples based on ASTM C273 without consideration of cell size effects.

\subsection{Shear Response - Analysis}

A model for the density dependence of the shear modulus of aluminum foam is presented by the authors in [17] and is summarized here. The model considers a transversely isotropic representative volume element (RVE) oriented in three-dimensional space. The constitutive properties of the RVE can take various forms such as a concentric cylinder consisting of a fiber surrounded by matrix material or a platelet reinforcement set in a matrix, as shown in Figure 6. The concentric cylinder may be used to model an open cell foam or the network of cell edges (cell edges are the intersection of cell walls) of a closed cell foam, which have been shown to dominate metal foam deformation [18]. The constitutive properties are evaluated to include cell wall imperfections such as curvature and corrugation, which couples bending and stretching deformation in the cell edges, also consistent with observations [18]. The magnitude of these imperfections and the geometry of the RVE are taken from measurements presented in [19].

The RVE is subjected to both a state of constant shear strain, following the method of [20], as well as a state of constant shear stress. In composite materials, a state of constant strain is an appropriate assumption when fibers are oriented parallel to the direction of loading, whereas a state of constant stress is appropriate when the fibers are oriented perpendicular to the direction of loading. For a foam, then, with its load bearing members oriented randomly in space, a stress state between these two extremes is appropriate. The assumption of constant stress results in the Reuss bound for the shear moduli and the assumption of constant strain results in the Voigt bound.

The Voigt bound is

$$
G_{\text {foam }}=\frac{\rho_{r} E_{s} I\left(65+4 \rho_{r} \nu_{f}-4 \rho_{r}^{2} \nu_{f}^{2}\right)}{90\left(1+\rho_{f} \nu_{f}\right)\left(2 I+A \sum_{n} a_{n}^{2}\right)}
$$

and the Reuss bound is

$$
G_{\text {foam }}=\frac{60 \rho_{r} E_{s} I}{\left(61+56 \rho_{r} \nu_{f}+4 \rho_{r}^{2} \nu f^{2}\right)\left(2 I+A \sum_{n} a_{n}^{2}\right)}
$$

in which the terms are defined as follows: $\rho_{r}$ is the relative density of the foam, $E_{s}$ is the uniaxial modulus of the foam's parent material, $I$ and $A$ are the 
cross-sectional properties of the cell edges, $\nu_{f}$ is the Poisson ratio of the foam, and $a_{n}$ are the magnitudes of the sinusoidal imperfections in the foam cell edges, magnitudes which were reported in [19].

Within the validity of its assumptions, the model produces an envelope of theoretically acceptable values for the shear modulus of the aluminum foam studied in the present work. The envelope and the experimental data presented above are plotted together in Figure 7. The model and the experimental data agree well. Also plotted is the lower bound of a theory that uses a cubic lattice structure RVE [5]

$$
G_{\text {foam }}=\frac{3}{8}(1.0-0.1) E_{s}\left[0.5 \rho_{r}^{2}+0.3 \rho_{r}\right]
$$

A proper extension of the theory presented here is to extend the model to include mechanismbased failure such that the entire shear response may be analyzed.

\section{$3 \quad$ Size Effects - Experiments}

The results presented above and in the related papers are bulk property measurements. In each work, the aluminum foam has been treated as a continuous, homogeneous medium, despite obvious inhomogeneities. Regardless of this fact, such a treatment is valuable if aluminum foam is to be integrated into engineered structures, in which prevailing analysis rests on these very assumptions. By treating the foam in this manner, congruent to our analysis of materials such as metals, woods, and polymers, it is possible to directly compare the performance of aluminum foam with other competitive engineering materials.

As metals are inhomogeneous on the microscopic level, comprised of individual crystals, metal foams are inhomogeneous on the cell level. When a sufficient amount of the inhomogeneous substructure is considered, the material acts as a homogeneous continuum. In the present work, experimental measurements related to the shear response are used to determine the length scale at which aluminum foam may be treated as a homogeneous continuum.

The square specimens that were tested in shear, as described above, were painted flat white and sprinkled with black glitter to form a random black and white pattern. As the specimen was deformed, high resolution black and white digital images (2028x2044 pixels) were captured incrementally. With Surface Displacement Analysis software from the Instron Corporation, the images were segmented into boxes of $32 x 32$ pixels. The software invokes digital image correlation to follow the movement of the black and white pattern in each box, outputting an average displacement vector for each box. This provides a displacement field for the entire surface of the sample.

Shear strains may be evaluated by considering square sub-regions of the sample, concentric with the sample itself, as is shown in Figure 8. The values for displacement of each point along the edge of the sub-region provide the deformation of the edge of the sub-region. By interpreting the change in angle between the edges of the sub-region from picture to picture, shear strains may be quantified incrementally, consistent with the definition of engineering shear strain [21]. In summing up each increment of shear strain, the state of strain in the sample is known at distinct points throughout the loading program.

Sub-regions of varying size, all concentric with the sample itself, are evaluated for shear strain. The size of the sub-region is expressed in mean cell diameters. Measurements of the mean cell diameters for a range of aluminum foam densities is available in [19] and is used here to define the mean cell diameter. The smallest sub-region shown in Figure 8, for example, has 9 mean cell diameters along each of its edges. The next larger sub-region is 12 mean cell diameters in size, and so on by three's.

With the strain known for each sub-region, the stress-strain response curve may be plotted for each sub-region, as is shown in Figure 9. The results clearly indicate that for sub-regions with dimensions shorter than 18 mean cell diameters, the local stressstrain response deviates significantly from the bulk material response. And for sub-regions with dimensions larger than 18 mean cell diameters, the response remains consistent with the bulk properties.

Figure 9 indicates a decline in material stiffness with decreasing sample size, but this is not a general result. Some specimens may even exhibit an increase in stiffness with decreasing sample size. As the sample size decreases, the mechanical behavior of individual cells is magnified as it is no longer averaged out by as many competing neighboring cells. The general result is that the uncertainty in the material response increases as the sample size decreases. This is the essence of mechanical behavior in material samples that are of a size compararble to or smaller than the characteristic dimension.

The experimental result is further illustrated in Figure 10, in which the ratio $\frac{\epsilon}{G_{b u l k}}$ is plotted as a 
percentage versus the number of cells in the subregion. $\epsilon$ represents the uncertainty in the shear modulus for a given sub-region. The uncertainty is defined as the difference between the shear modulus of the sub-region and the shear modulus of the bulk material, $G_{b u l k}$. As the number of cells increases, the ratio reaches a value of less than $5 \%$ at a sub-region size of 18 cells and remains small and constant for all sub-regions of larger dimensions.

The result presented here compares favorably with results for other macroscopically inhomogeneous materials. [22], [23], and [24] each found that size effects in reticulated foams dominated the sample response if the critical dimension of the sample measured fewer than 20 mean cell diameters [2]. Furthermore, the strength of the sub-regions represented in Figure 9 does not decline, consistent with the results of [25], in which shear strength of metal foam samples did not decline until the sample dimensions were less than 3 mean cell diameters.

Optical strain measurement is an ideal method for obtaining size effects for two reasons. First, a broad range of sample sizes may be tested simultaneously and, therefore, subjected to identical test conditions. Second, the ratio of surface area to volume is unchanged. When the samples are machined, cells on the sample edge are damaged. The mechanical integrity of these cells is diminished, yet they still contribute to the overall volume of the sample. The ratio of the damaged edge cells to undamaged cells increases as the sample size decreases. In the present method, this ratio remains constant, allowing for the testing of small samples that are free of cells damaged by cutting.

\subsection{Size Effects - Analysis}

The subject of homogenization of inhomogeneous materials is a complex topic that requires a rigorous mathematical approach to obtain a true understanding of its related nuances. Such approaches are summarized in [26] and [27]. The present approach is an attempt to illustrate and replicate with a simple, sensible model the size effect behavior observed in the experiments.

Aluminum foam has been modeled as a random structure and as a periodic structure; its cells have been considered perfect as well as imperfect. In [17], as summarized above, the foam is modeled as a random structure with imperfections. In [2] and [25], among others, it is modeled as a perfect periodic structure. Even though simple observation reveals that aluminum foam is an imperfect, semi- random structure, the results of the previous section illustrate that the random and imperfect nature of the foam structure is important in shear only for samples with dimensions fewer than 18 mean cell diameters. For a sample smaller than the threshold value, the shear response depends highly on the specific imperfections contained within the sub-region. Some imperfections act as stiffening mechanisms, while others promote compliance. As the sample size decreases, imperfections dominate and the uncertainty in its material response increases.

In the present analysis, a periodic structure of $N x N$ cells with randomly distributed imperfections is created and analyzed with finite elements. The structure is not meant to model aluminum foam. It is used as a tool to understand the behavior of periodic structures in shear as imperfections are introduced. The uncertainty in shear stiffness of the imperfect structures is of primary concern. The model will be used to observe the disappearance of this uncertainty as the sample size grows (as the number of cells, $N$, increases).

A $10 x 10$ imperfect periodic structure is shown in Figure 11, as an example. The structure is made of four basic unit cells, one perfect and three imperfect (Figure 12), randomly distributed, each with an equal probability of inhabiting any given cell location. There are $100\left(N^{2}\right)$ cell locations. The structure is sheared and the shear stiffness is computed. The structure is then given a different cellular distribution using the same four basic cells and the shear stiffness is measured. This is repeated at least three times for each value of $N$ chosen from the range $3 \leq N \leq 50$. For a given value of $N$, multiple runs, each with its associated imperfect cell distribution, produce a range of shear stiffnesses. The range is taken to be the uncertainty, $\epsilon$, in the shear stiffness of the $N x N$ periodic structure. It was found that no more than three runs for each value of $N$ was necessary to produce a representative range of shear stiffness values.

The models were created and solved in ABAQUS with B23 cubic beam elements with solid rectangular cross-sections. The dimensions of the structure were scaled such that the perfect structure had a constant shear stiffness regardless of the value of $N$.

The ratio $\frac{\epsilon}{G_{\text {perfect }}}$ is plotted versus the number of cells $N$ in Figure 13, in which $G_{\text {perfect }}$ is the shear stiffness of the perfect periodic structure shown in Figure 14. The histogram in Figure 13 is remarkably similar to that in Figure 10. For structures with greater than 18 cells in each dimension, 
the uncertainty in shear stiffness becomes small and remains relatively constant for all larger specimen sizes.

A pertinent response to this result is to consider the degree of imperfection introduced in the above model. Perhaps this set of allowable imperfections is such that the uncertainty in shear stiffness just so happens to drop off for sample dimensions larger than 18 cells. Perhaps a more detrimental set of imperfections (i.e.- imperfections that reduce the shear stiffness of a given sample more drastically than those chosen here) would require even larger sample sizes before the uncertainty in shear stiffness approaches a constant value.

To investigate such a possibility, each beam member of the perfect structure shown in Figure 14 is given a $50 \%$ probability of disappearing from the structure entirely. In the previous problem only the cross bars (the diagonal elements of each cell) were given a $50 \%$ probability of disappearing, while the horizontal and vertical cell members always remained. An example of the newly-degraded structure is shown in Figure 15.

The same procedure of introducing three sets of imperfections into each $N x N$ sample is followed and the results are plotted in Figure 16. Clearly, despite the drastic increase in imperfection population, the uncertainty in the shear stiffness of the structure still approaches a constant value when the specimen dimensions are greater than 18 cells.

Structures of different geometry (i.e.- honeycombs with circular, hexagonal, or triangular cells) and varying degrees of imperfection would be valuable subjects of study in order to understand the dependence, if any, of the results presented here on such parameters. The only size effect studies regarding aluminum foam known to the authors are found in [25] and [28]. In [25], the shear modulus of an analytical regular hexagonal honeycomb structure is analyzed by equilibrium and is shown to converge to have bulk shear stiffness when the number of cells is three or greater through the thickness. Since the model contains perfect cells, the convergence properties do not represent the averaging out of imperfections, as are present in metal foams. Rather, the convergence depends solely on the shape of the cell chosen, in this case, hexagonal honeycomb. In [28], aluminum foam specimens of varying thickness are sheared experimentally. It is found that the strength of these foams does not depend on specimen thickness for specimens with at least three cells through the thickness. The results of the present study are consistent with [28]. As illustrated in Figure 9, a loss of strength is not observed for any of the specimens, each of which is larger than three cell sizes.

\section{Conclusions}

The results presented in the present paper are intended to reveal the characteristics of aluminum foam that are of primary concern for sandwich structure cores. The shear response was measured and shown to have an initial linear region accompanied be increasing plastic strain even at low load levels. After peak load, the load carrying capability of the foam dropped off quickly. Up to low-rate dynamic loading, the linear response was independent of strain rate while the peak load and energy absorption each increased as the strain rate increased. A model based on the composite cylinders model simulated the network of imperfect cell edges and offered an envelope of predicted values for the aluminum foam shear modulus.

Size effects were studied through experiment and simulation. Digital image correlation provided the full displacement field on the surface of the foam throughout the experimental loading program. Shear strain was calculated over concentric subregions of varying size within the sample. For subregions with dimensions shorter than 18 mean cell diameters, the shear response became uncertain. This uncertainty increased as the size of the subregion decreased. The same behavior was observed through a finite element model of a periodic structure with randomly distributed imperfections. For structures with dimensions shorter than 18 cells, the uncertainty in the shear response increased as the sample size decreased. This behavior was constant for various degrees of cellular imperfection.

\section{References}

[1] Rakow, J.F, Waas, A.M. Integrally bonded sandwich structures. In submission.

[2] Gibson, L.J, Ashby, M.F. Cellular Solids: Structure and Properties. Cambridge: Cambridge University Press, 1997

[3] Final Report of the X-33 Liquid Hydrogen Tank Test Investigation Team, Marshall Flight Center. Huntsville, AL, May 2000

[4] von Hagen, H, Bleck, W. Compressive, tensile, and shear testing of melt-foamed aluminum. Mat. Res. Soc. Symp. Proc, Vol.521, 1998, p.59 
[5] Ashby, M.F, Evans, A.G, Fleck, N.A, Gibson, L.J, Hutchinson, J.W, Wadley, H.N.G. Metal Foams: A Design Guide. Boston: ButterworthHeinemann, 2000

[6] Saenz, E, Baranda, P.S, Bonhomme, J. Shear properties of aluminum metal foams prepared by the melt route. Mat. Res. Soc. Symp. Proc, $\mathrm{V}$

[7] Rakow, J.F, Waas, A.M. Size effects and the shear response of aluminum foam. In submission.

[8] Dannemann, K.A, Lankford Jr, J. High strain rate compression of closed-cell aluminum foams. Mat. Sci. Eng. A, Vol.293, 2000, p.157

[9] Hall, I.W, Guden, M, Yu, C.-J. Crushing of aluminum closed cell foams: density and strain rate effects. Scripta Mater, Vol.43, 2000, p.515

[10] Paul, A, Ramamurty, U. Strain rate sensitivity of a closed-cell aluminum foam. Mat. Sci. Eng. A, Vol.281, 2000, p.1

[11] Mukai, T, Kanahashi, H, Miyoshi, T, Mabuchi, M, Nieh, T.G, Higashi, K. Experimental study of energy absorption in a close-celled aluminum foam under dynamic loading. Scripta Mater, Vol.40, No.8, 1999, p.921

[12] Deshpande, V.S, Fleck, N.A. High strain rate compressive behaviout of aluminum foams. Intl. J. Impact Eng, Vol.24, 2000, p.277

[13] McCullough, K.Y.G, Fleck, N.A, Ashby, M.F. Uniaxial stress-strain behaviour of aluminum alloy foams. Acta Metall, Vol.47, No.8, 1999, p. 2323

[14] Andrews, E, Sanders, W, Gibson, L.J. Compressive and tensile behaviour of aluminum foams. Mat. Sci. Eng. A, Vol.270, 1999, p.113

[15] Motz, C, Pippan, R. Deformation behaviour of closed-cell aluminum foams in tension. Acta Mater, Vol.49, 2001, p.2463

[16] Bastawros, A-F, Bart-Smith, H, Evans, A.G. Experimental analysis of deformation mechanisms in a closed-cell aluminum alloy foam. J. Mech. Phys. Solids, Vol.48, 2000, p.301

[17] Rakow, J.F, Waas, A.M. On the elastic moduli of random fibrous composites, platelet composites, and foamed solids. Accepted to appear in Mechanics of Advanced Materials and Structures, 2003.
[18] Bart-Smith, H, Bastawros, A.-F, Mumm, D.R, Evans, A.G, Sypeck, D.J, Wadley, H.N.G. Compressive deformation and yielding mechanisms in cellular $\mathrm{Al}$ alloys determined using x-ray tomography and surface strain mapping. Acta Metall, Vol.46, No.10, 1998, p.3583

[19] Simone, A.E, Gibson, L.J. Aluminum foams produced by liquid-state processes. Acta Mater, Vol.46, No.9, 1998, p.3109

[20] Christensen, R.M, Waals, F.M. Effective stiffness of randomly oriented fibre composites. J. Comp. Mat, Vol.6, 1972, p.518

[21] Fung, Y.C. Foundations of Solid Mechanics. Englewood-Cliffs: Prentice-Hall, 1965

[22] Lakes, R.S. Size effects and micromechanics of a porous solid. J. Mat. Sci, Vol.18, 1983, p.2572

[23] Brezny, R, Green, D.J. The effect of cell size on the mechanical behavior of cellular materials. Acta Metall. Mater, Vol.38, No.12, 1990, p.2517

[24] Mora, R. Waas, A.M. Strength scaling of brittle graphitic foam. Proc. Royal Soc: A, Vol.458, 2002, p.1695.

[25] Onck, P.R, Andrews, E.W, Gibson, L.J. Size effects in ductile cellular solids. Part I: modeling. Intl. J. Mech. Sci, Vol.43, 2001, p.681

[26] Manevich, L.I. Mechanics of Periodically Heterogeneous Structures. New York: Springer, 2002

[27] Cioranescu, D. Homogenization of reticulated structures. New York: Springer-Verlag, 1999

[28] Andrews, E.W, Gioux, G, Onck, P, Gibson, L.J. Size effects in ductile cellular solids. Part II: experimental results. Intl. J. Mech. Sci, Vol.43, 2001, p.701 
AIAA-2003-1946

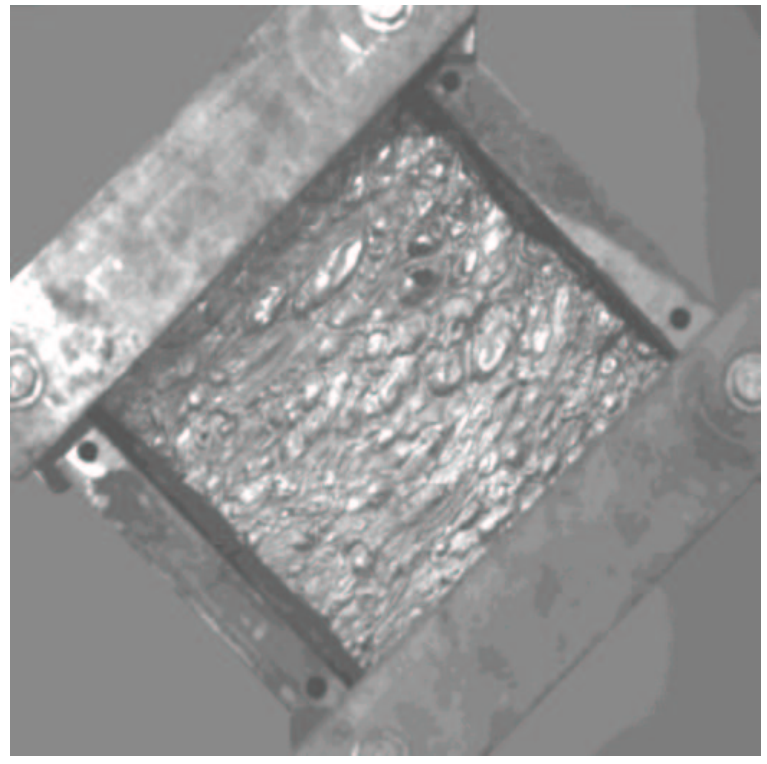

Figure 1: The loading frame used in shear testing of aluminum foam

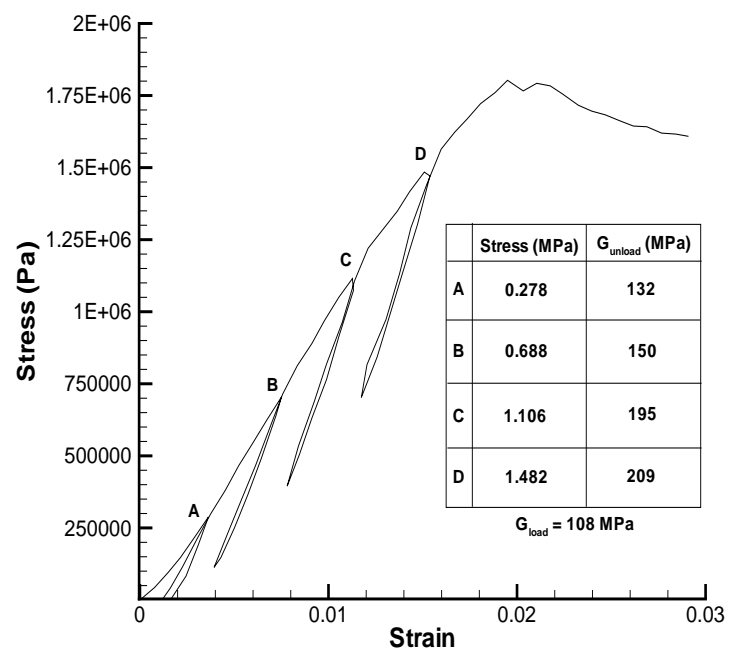

Figure 2: Representative shear response curve with unloading behavior. Plastic strain exists at very low load levels and increases throughout the linear region, stiffening the unloading response.

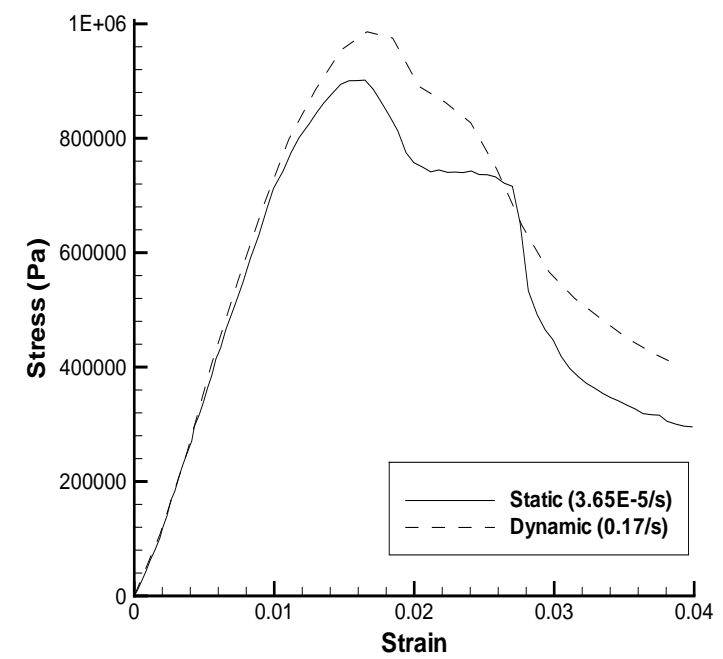

Figure 3: Comparison between the static and dynamic response curves for two samples of equivalent density

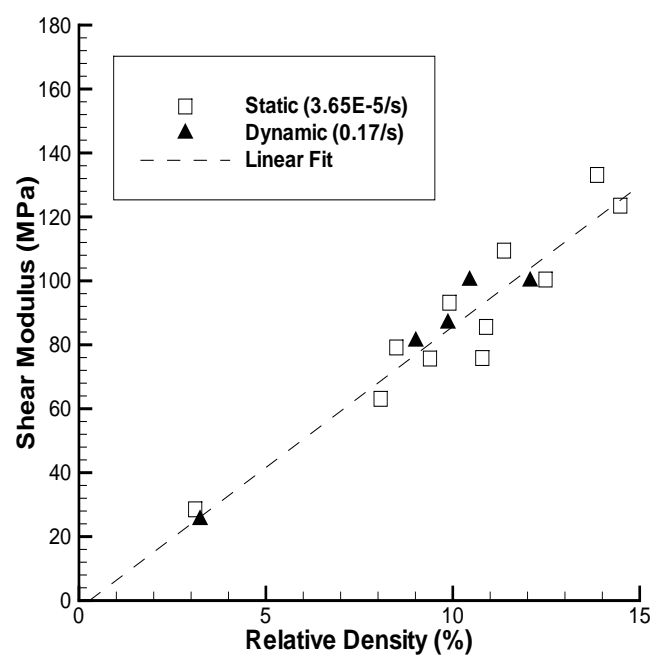

Figure 4: Shear modulus versus density for static and dynamic loading. No rate effects are observed 


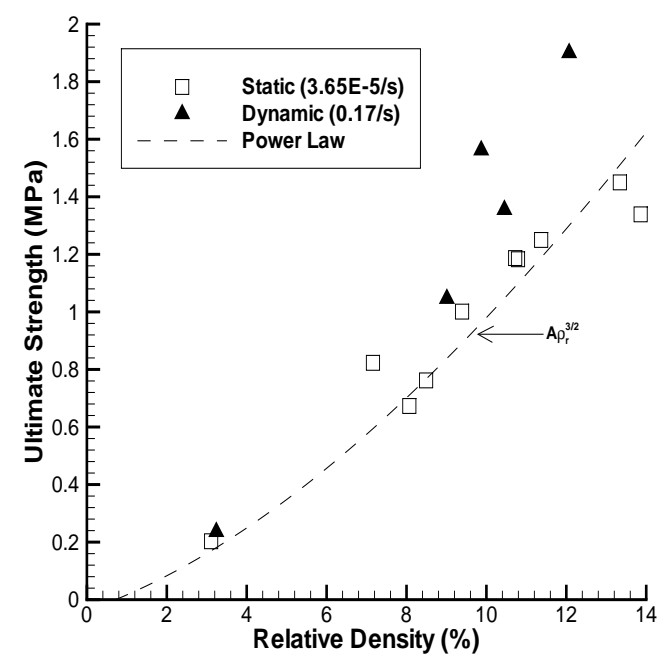

Figure 5: Shear strength versus density for static and dynamic loading. Rate effects are observed.

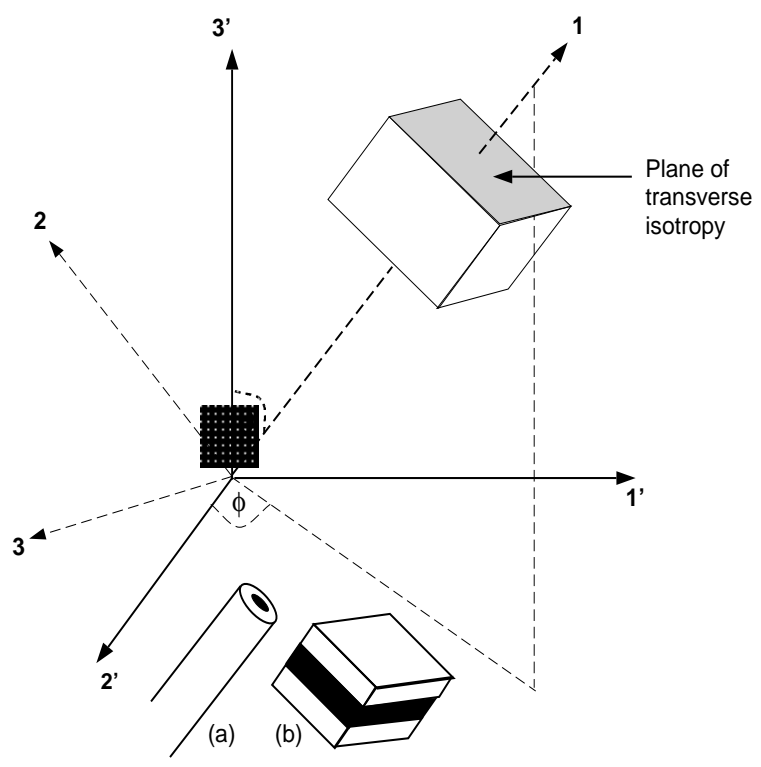

Figure 6: A transversely isotropic RVE oriented in three-dimensional space. The RVE may be (a) a concentric cylinder or (b) a platelet-matrix layering, as shown

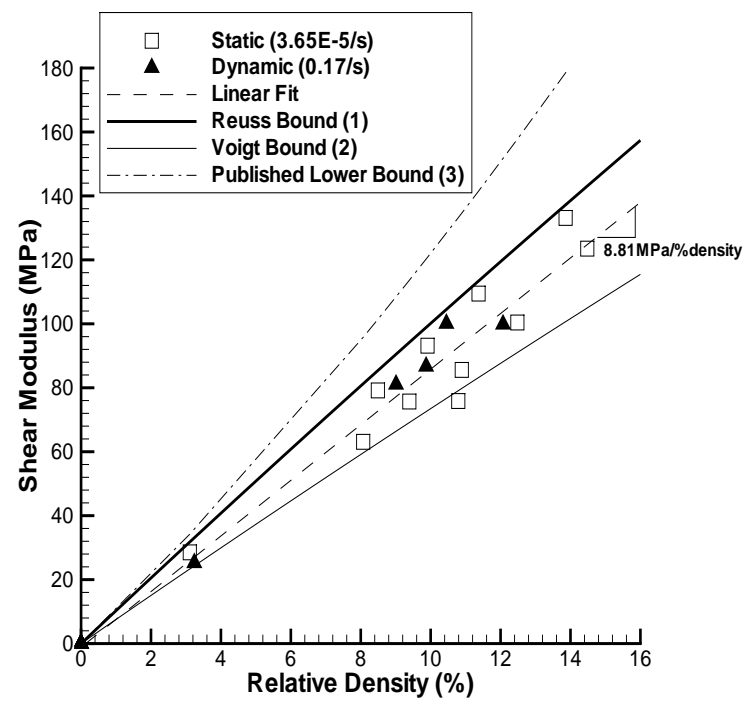

Figure 7: The model produces appropriate bounds for the experimental shear moduli.

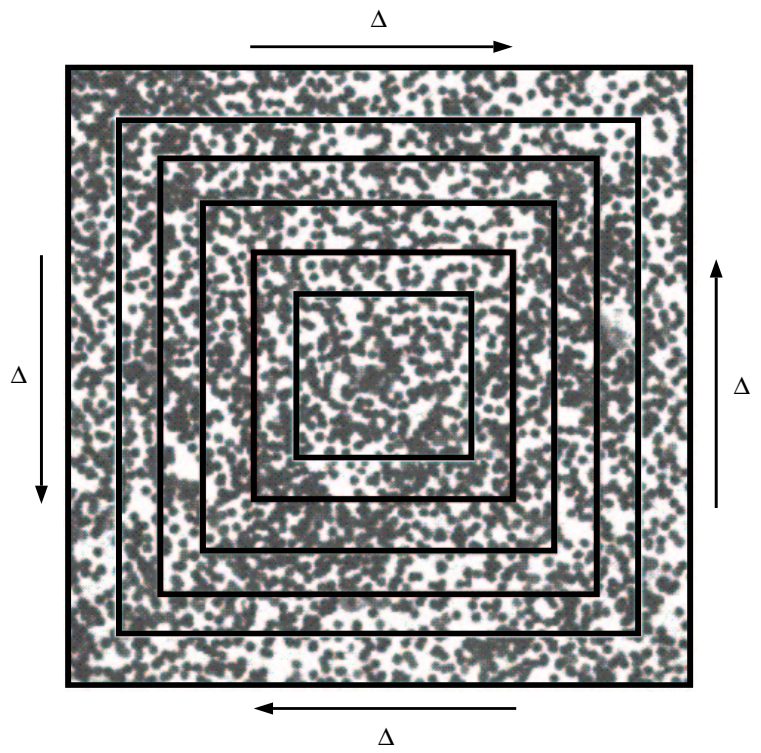

Figure 8: The black and white patterned specimen with its concentric sub-regions over which shear strain is calculated. The black lines represent the boundaries of the sub-regions. 


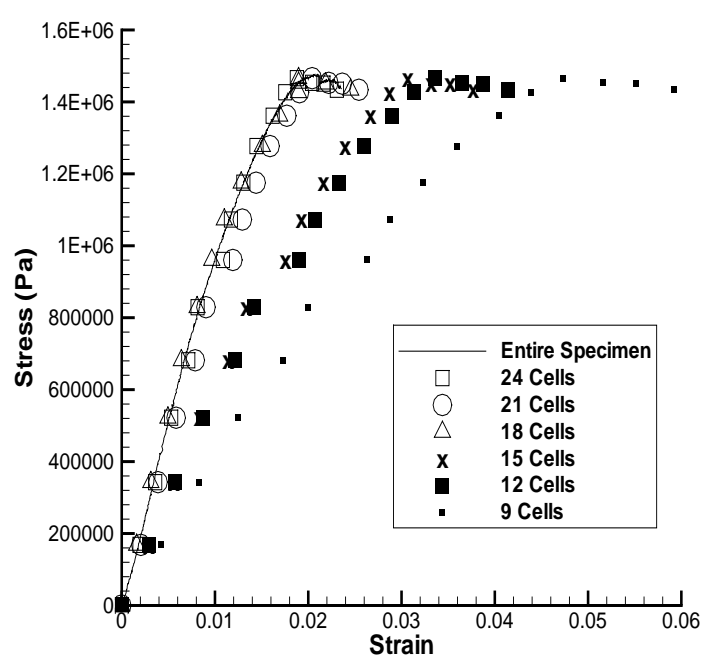

Figure 9: The shear response of various sub-regions within a given sample

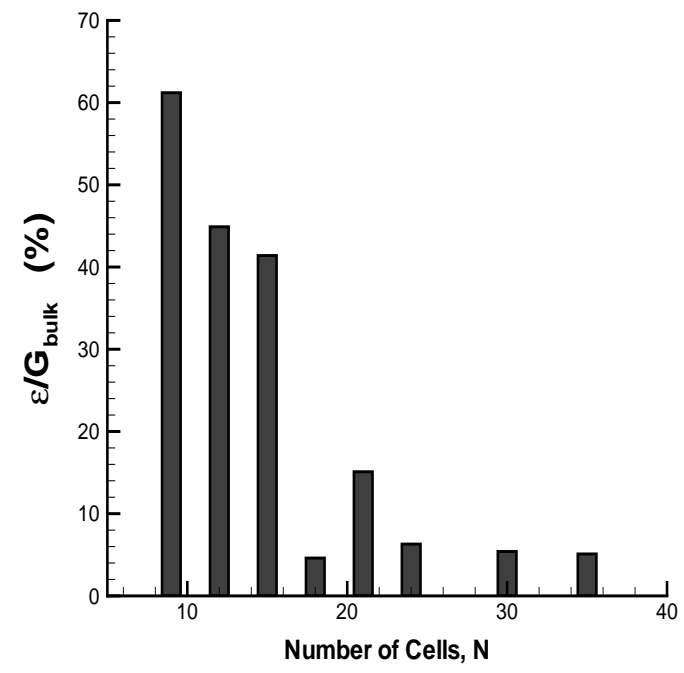

Figure 10: The shear modulus of the sub-regions converges to that of the bulk sample as the size of the sub-region approaches 18 mean cell diameters.

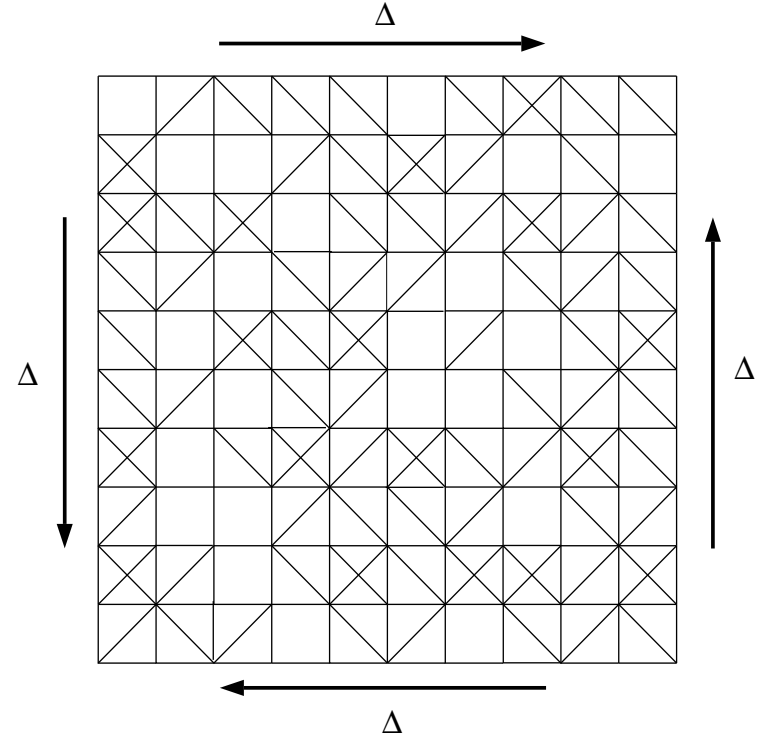

Figure 11: The finite element model of a $10 x 10 \mathrm{im}-$ perfect periodic structure. The geometry of each cell is chosen randomly from the four cells in Figure 12

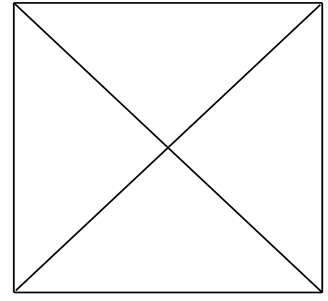

Perfect

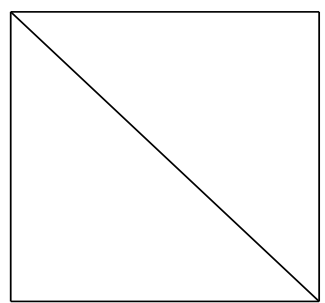

Imperfect

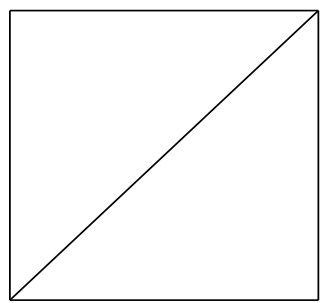

Imperfect

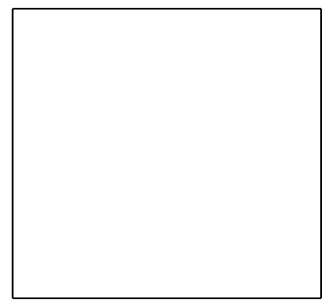

Imperfect

Figure 12: The four cells that are used to construct a periodic structure with randomly distributed imperfections 


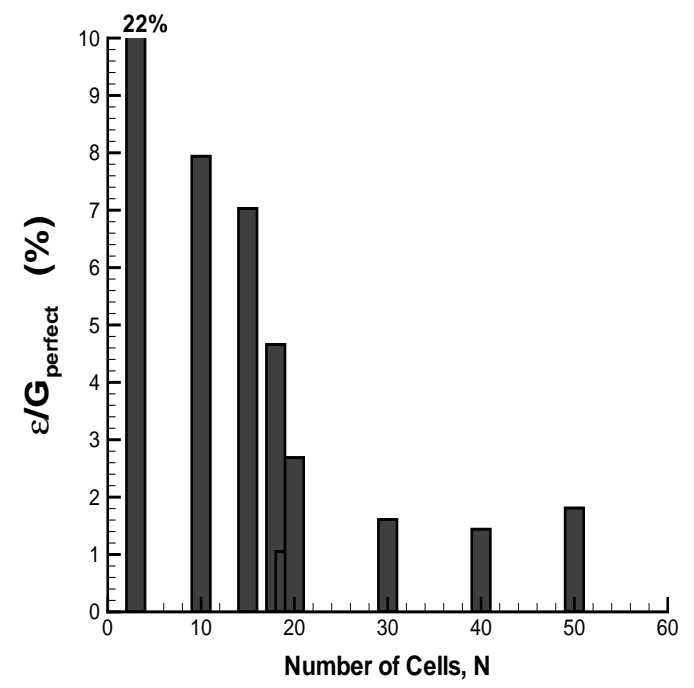

Figure 13: The uncertainty in the shear stiffness of the imperfect structure in Figure 11 approaches a constant value for a structure larger than $18 \times 18$ cells.

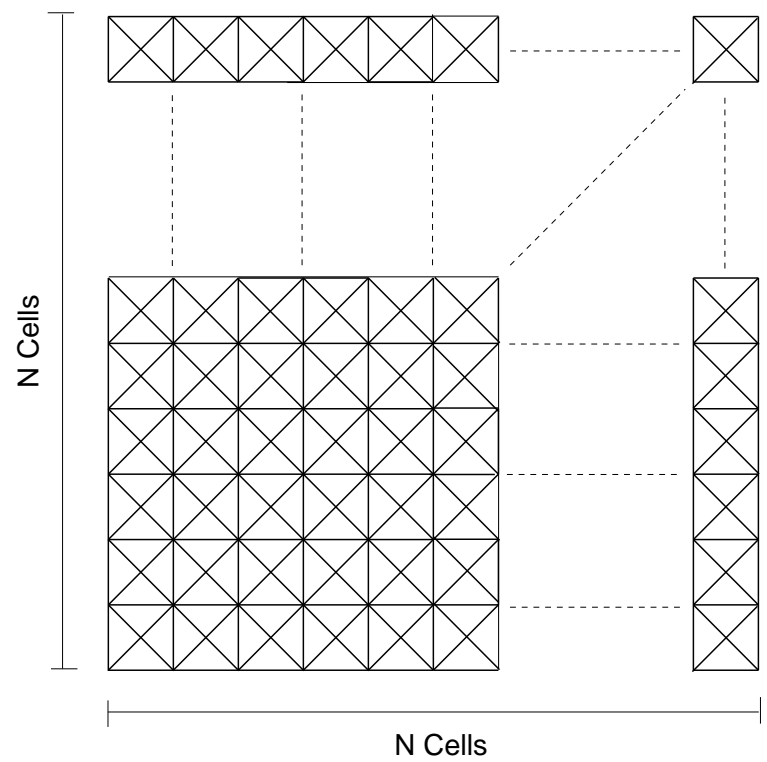

Figure 14: The perfect periodic structure has $N x N$ perfect cells.

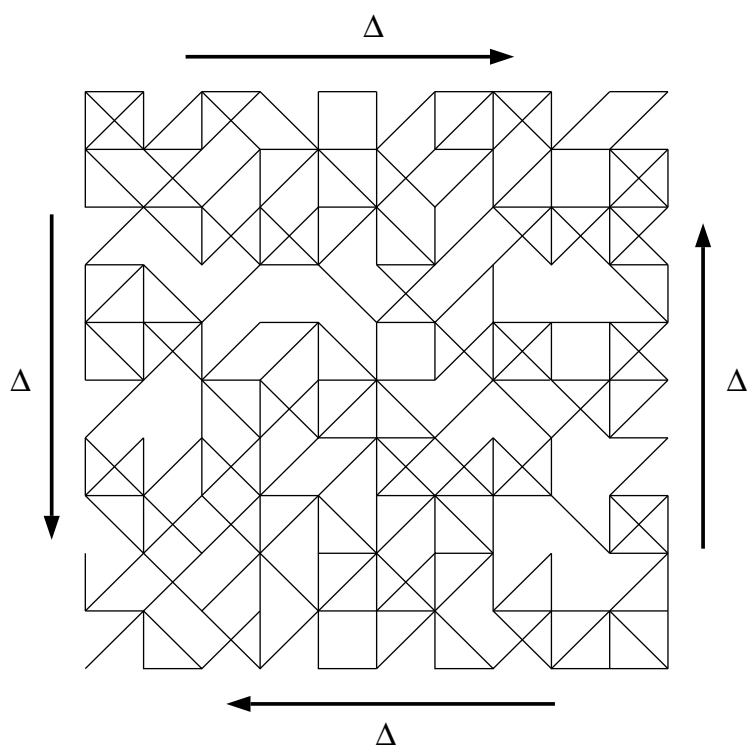

Figure 15: The finite element model of an imperfect periodic structure in which the imperfections are such that any member of any cell has a $50 \%$ probability of being non-existent

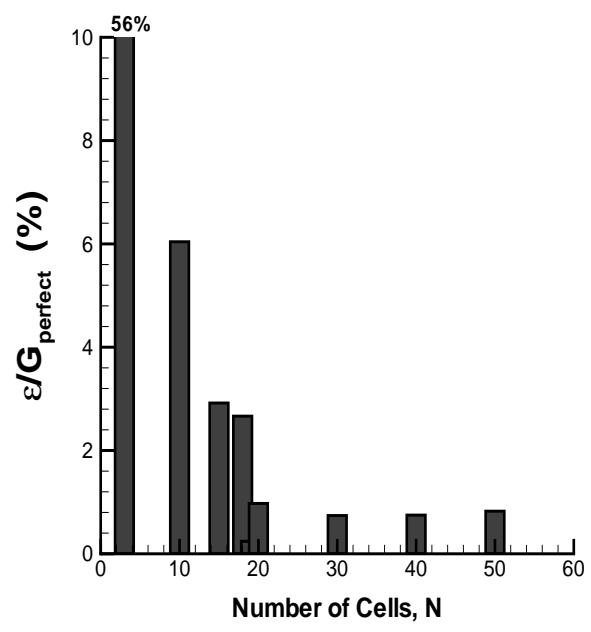

Figure 16: The uncertainty in the shear stiffness of the imperfect structure in Figure 15 approaches a constant value for a structure larger than $18 \times 18$ cells. 